\title{
Aptamer-Based Surface Plasmon Fibre Sensor for Thrombin Detection
}

\author{
T. Allsop, D. Nagel ${ }^{1}$, R. Neal ${ }^{3}$, E. M. Davies, C. Mou, P. Bond ${ }^{4}$, S. Rehman ${ }^{6}$, K. Kalli ${ }^{2}$, D.J. Webb \\ P. Calverhouse ${ }^{3}$, M. Mascini ${ }^{5}$, I. Bennion \\ Authors' Affiliations \\ Photonics Research Group, Aston University, Aston Triangle, Birmingham, B47ET, UK. \\ ${ }^{1}$ Dept. Life and Health Sciences, Aston University, Aston Triangle, Birmingham, B47ET, UK. \\ ${ }^{2}$ Cyprus University of Technology, 31 Archbishop Kyprianos, Lemessos 3036, Cyprus \\ ${ }^{3}$ Dept of Maths and Computing, and the Faculty of Science and Technology, University of \\ Plymouth, Plymouth, PL4 8AA, U.K. \\ ${ }^{4}$ Plymouth Electron Microscopy Unit, University of Plymouth, Plymouth, PL4 8AA, U.K \\ ${ }^{5}$ Dipartimento di Chimica, Università di Firenze, Via della Lastruccia 3, 50019 Sesto Fiorentino, \\ Italy \\ ${ }^{6}$ STR Fiber Technologies, Rickmansworth, Herts, UK
}

\begin{abstract}
A series of surface plasmonic fibre devices were fabricated using multiple coatings deposited on a lapped section of a single mode fibre and post-fabrication UV laser irradiation processing with a phase mask, producing a surface relief grating structure. These devices showed high spectral sensitivity in the aqueous index regime ranging up to 4000 $\mathrm{nm} / \mathrm{RIU}$ for wavelength and $800 \mathrm{~dB} / \mathrm{RIU}$ for intensity. The devices were then coated with human thrombin binding aptamer. Several concentrations of thrombin in buffer solution were made, ranging from $1 \mathrm{nM}$ to $1 \mu \mathrm{M}$. All the concentrations were detectable by the devices demonstrating that sub-nM concentrations may be monitored.
\end{abstract}

Keywords: Bio-sensing, surface plasmons, fibre optic sensors, thrombin

\section{Introduction}

As biotechnology progresses, aptamers have come to the fore as attractive diagnostic reagents and potential antibody replacements for the development of biomolecular nanosensors due to their high affinity, specificity, and stability ${ }^{1-4}$ and the fact that they can be synthesized in vitro. One application for aptamers is the measuring the levels thrombin, important in the blood coagulation process. Several types of tests have been developed to quantify thrombin, such as end-point assays measuring the formation of clots or chromogenic and fluorescent substrates ${ }^{5}$ that allow thrombin activity to be detected through the use of spectrophotometers. Fibre optic evanescent wave sensors based upon fluorescent coagulation ${ }^{6}$ and other evanescent wave sensors, such as surface plasmon resonance sensors ${ }^{7-9}$, have been developed recently. These have been used to detect other biochemical compounds by utilising immobilized biorecognition molecular coatings on a metallic surface.

Surface plasmon resonance is an important optical phenomenon that involves the resonant transfer of incident propagating light to surface-plasmon modes that takes the form of collective electron oscillations in a metal ${ }^{10}$. Sensors based on surface plasmon resonance (SPR) have distinct advantages over conventional fibre sensors that are based upon gratings, the principal advantage being high spectral sensitivity. These sensors can be divided into three types based upon the kind of surface plasmon being generated at the metal/dielectric interface: the long range surface plasmon

Biophotonics: Photonic Solutions for Better Health Care II, edited by Jürgen Popp,

Wolfgang Drexler, Valery V. Tuchin, Dennis L. Matthews, Proc. of SPIE Vol. 7715, 77151C

(C) 2010 SPIE · CCC code: $1605-7422 / 10 / \$ 18 \cdot$ doi: $10.1117 / 12.854334$

Proc. of SPIE Vol. $771577151 \mathrm{C}-1$ 
$(\text { LRSP) })^{11}$, the short range surface plasmon (SRSP) ${ }^{12}$ and the localised surface plasmon ${ }^{13}$. It has been found that SPR generation is very sensitive to the polarisation of the illuminating light, its wavelength and its angle of incidence on the metal surface. This polarisation sensitivity can be used to detect index changes in biochemical/chemical reactions ${ }^{14}$. SPR biosensors offer the opportunity for real-time and label-free monitoring of biomolecular interactions ${ }^{15}$. The plasmons exist at a metal-dielectric interface and depending on the topology of supporting metal coating the dispersion relation can change ${ }^{13}$; the LRSP and SRSP obey the following dispersion relation for two homogeneous semi-infinite media:

$$
\beta=k \sqrt{\left(\frac{\varepsilon_{m} \cdot n_{s}{ }^{2}}{\varepsilon_{m}+n_{s}{ }^{2}}\right)}
$$

where $k$ is the free space wave number, $\varepsilon_{m}$ is the dielectric constant of the metal $\left(\varepsilon_{m}=\varepsilon_{m r}+i \varepsilon_{m i}\right)$ and $n_{s}$ is the refractive index of the dielectric sample to be tested.

\section{Fabrication and Characterisation of SPR Devices}

The fibre devices were constructed in three stages ${ }^{15}$. Firstly, a standard single mode fibre (Corning, SMF-28e) was mechanically lapped down to within $10 \mu \mathrm{m}$ of the core-cladding interface. Secondly, using RF sputtering, a series of coatings were deposited upon the flat of the lapped fibre. These coatings consisted of germanium $(48 \mathrm{~nm})$, silicon dioxide $(48 \mathrm{~nm})$ and gold $(38 \mathrm{~nm})$,. The coated fibre was then exposed to a UV light interference pattern produced with a uniform phase mask of period $1.018 \mu \mathrm{m}$, via laser beam scanning and multi-exposures. This produced a surface relief structure with dominant spatial periods close to $0.5 \mu \mathrm{m}, 1 \mu \mathrm{m}, 2 \mu \mathrm{m}$ and $4 \mu \mathrm{m}$. The devices were characterised by measuring the changes in their response as a function of the polarisation properties of the illuminating light and the changes in the surrounding medium's refractive index. Light from a broadband light source, was passed through a polariser and a polarisation controller before illuminating the sample, with the transmission spectrum being monitored using an optical spectrum analyser. The change in polarisation of the illuminating light is monitored with a polarimeter (Tektronix, PAT 9000B) through a polarisation maintaining coupler (fig. 1). Polarisation sensitivity results are shown in figure 2.

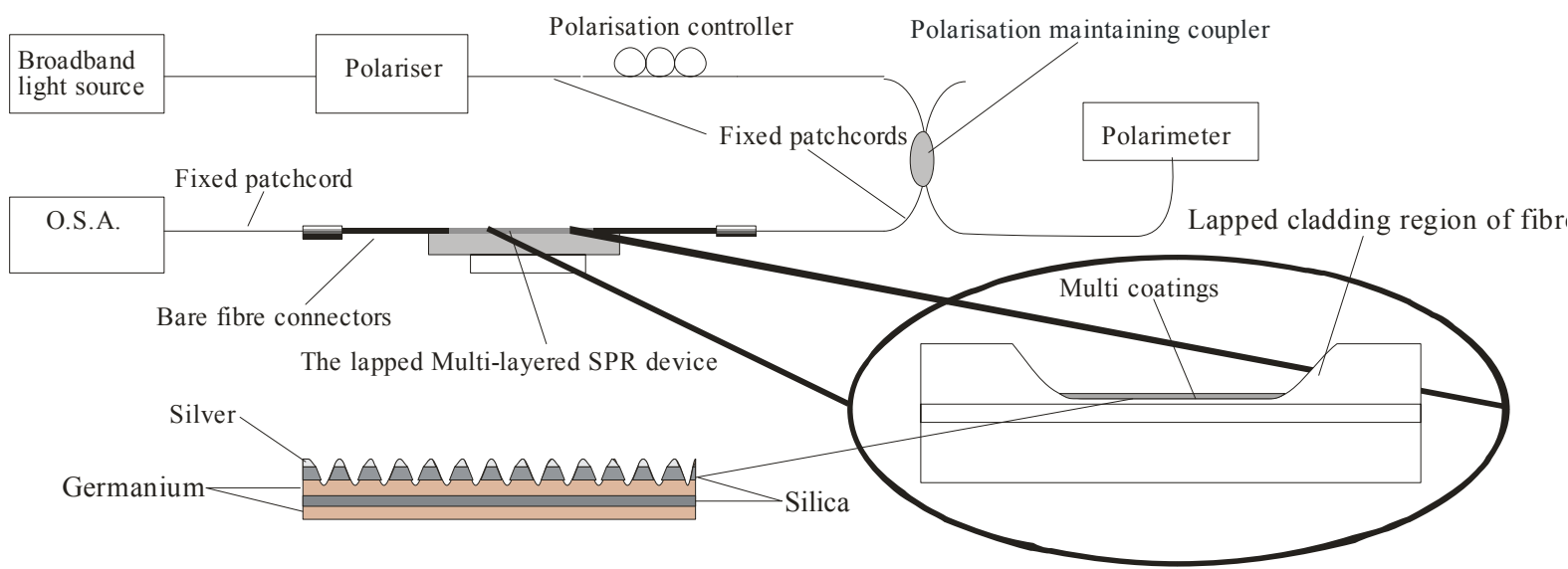

Figure 1. Scheme used for the characterisation of the devices

The three devices are polarisation dependent with multiple resonances at different wavelengths observed with the surrounding medium having an index of 1.36 , see figure 1 for examples. 


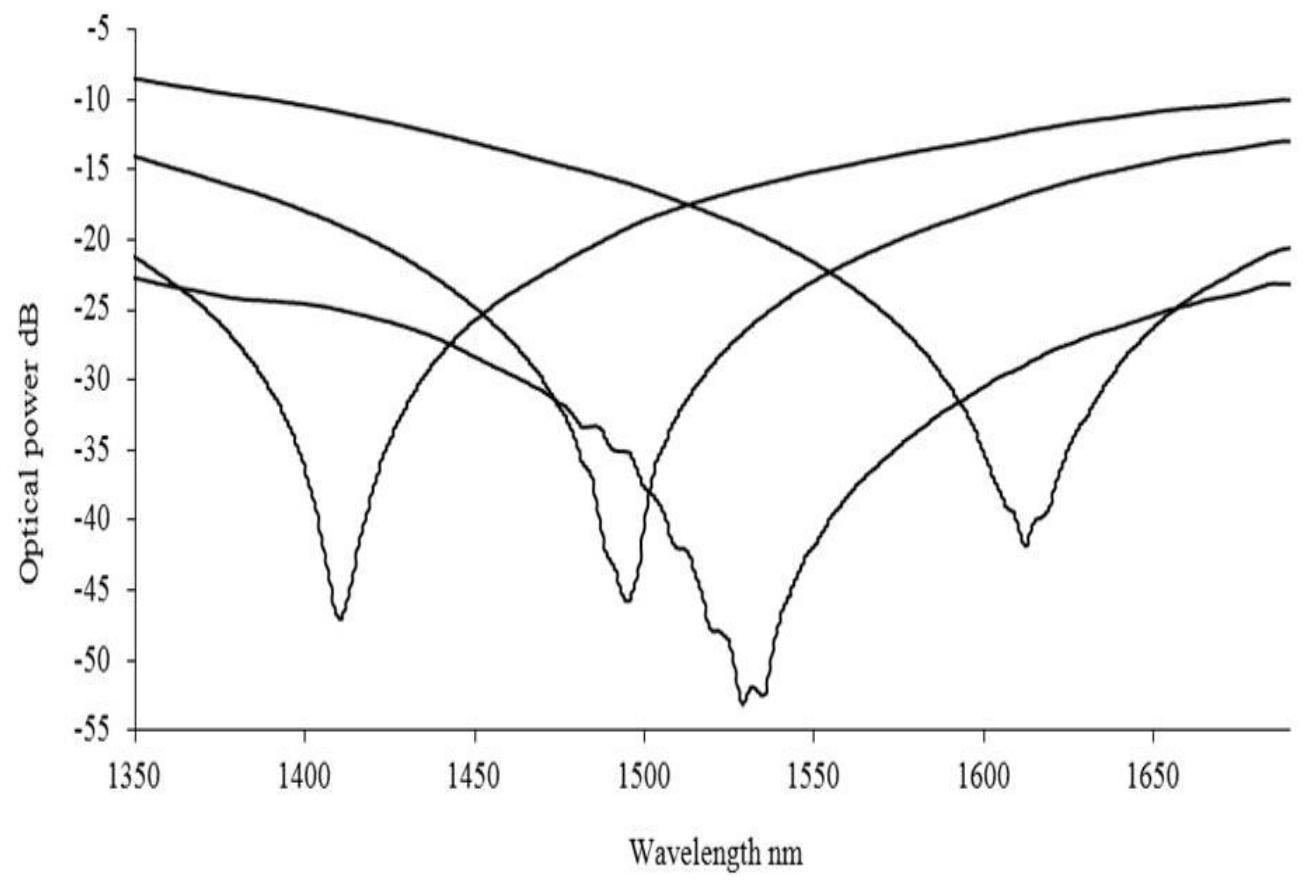

Figure 2 The observed surface plasmon resonances obtained at different wavelength by varying the polarisation of the illuminating light for the device consisting of $\mathrm{Au}-\mathrm{SiO}_{2}-\mathrm{Ge}$ with a surrounding refractive index of 1.36 .

\section{Aptamer thrombin assay procedure}

Coated fibres bearing the thrombin aptamer 5' - SH(CH2)6 - GGT TGG TGT GGT TGG - 3' imobilised to the fibre by a thiol mediated linkage (Marco could give this a proper name I presume it is a malamide coupling but don't want to guess) were a gift from / were obtained from ---- (there probably is a paper reference for this asptamer which could be included in the introduction). Fibres were stored in potassium phosphate buffer (100mM pH7.4). Alpha thrombin from human plasma was obtained from Sigma (Sigma T6884). Binding buffer (50mM Tris $140 \mathrm{mM} \mathrm{NaCl} 1 \mathrm{mM} \mathrm{MgCl} 2) \mathrm{pH}$ 7.4, was used for all binding experiments.

\section{Detection of thrombin}

Thrombin solutions ranging from $1 \mathrm{nM}-1 \mu \mathrm{M}$ were prepared in a binding buffer. The sensor was exposed to the solutions lying in a flat v-groove on an aluminum block. After each thrombin solution was used the v-groove was cleaned using a $50 \mathrm{mM} \mathrm{HCl}$ then washed with binding buffer. Due to the spectral broadness of these transmission features, the central wavelength was calculated by the first moment of the power spectrum; the centroid by geometric decomposition. The associated centroid strength value was obtained by the mean value over the interval range of interest. The optical power and wavelength shift results for the $\mathrm{Au}-\mathrm{SiO}_{2}-\mathrm{Ge}$ coated aptamer device are shown in figures 3 and 4. 

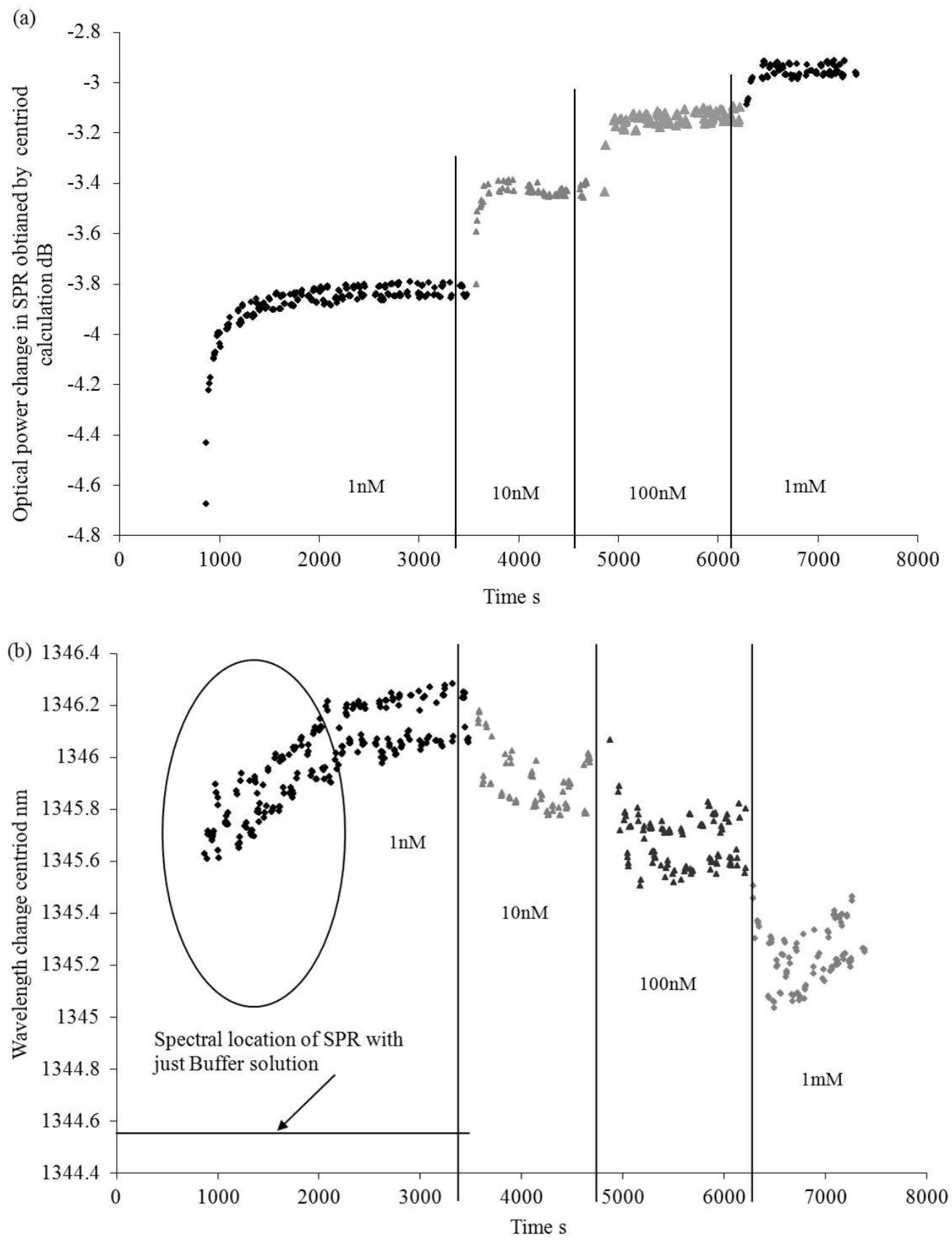

Figure 3 Experimental data for solutions of thrombin (1nM, 10nM, 100nM, 1mM) with respect to time taken for experiment, (a) the change in the optical strength of the SPR ( b) observed wavelength shift of the SPR, taking into account the buffer solution 

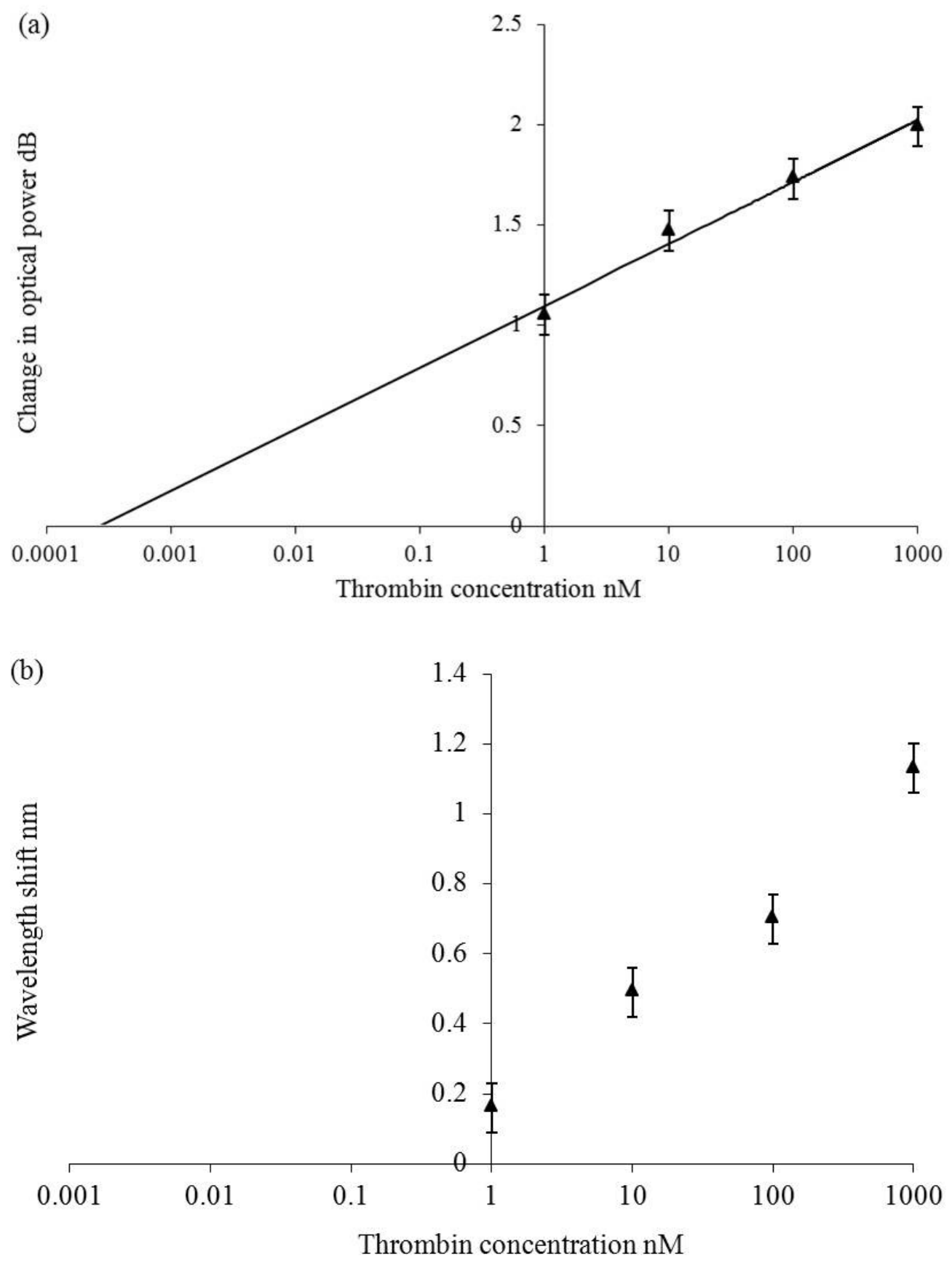

Figure 4 The spectral sensitivity of the aptamer coated Multilayered SPR fibre device as a function of thrombin concentration (a) the change in optical coupling strength (b) the change in centroid wavelength

Inspecting figure $3 \mathrm{~b}$ it is noticeable that for the $1 \mathrm{nM}$ concentration we observe a red wavelength shift (circled in the figure) whilst with the rest of the solutions the SPR fibre device yields a blue wavelength shift. The initial response may 
be showing the ingression of just the buffer solution into the coating. Secondly the wavelength response is not as clear as the optical power change, though we believe this can be improved by obtaining a more well-defined SPR coupling spectral feature. Assuming that the red wavelength shift is due to buffer ingression into the aptamer coating we can estimate the spectral and intensity sensitivity of the SPR device, see figure 4 . Working to the intensity detectability associated with this interrogation scheme $(0.1 \mathrm{~dB})$ and from the experimental data using linear regression, the empirical relationship $\Delta \mathrm{I}(\mathrm{dB})=0.1338 \mathrm{In}\left(\mathrm{Con}_{\mathrm{cen}}\right)+1.098$ was used to estimate the minimum detectable concentration of thrombin which is $500 \mathrm{fM}$. The same resolution allows us to detect a change in concentration of around $5 \%$, i.e. $50 \mathrm{pM}$ at $1 \mathrm{nM}$. We believe that these devices are creating localised surface plasmons (LSP), with decay length of the order of $\sim 20 \mathrm{~nm}$ (work to be published). For the following reason an adsorbed film of biomolecules (typically $<10 \mathrm{~nm}$ ) will occupy a larger fraction of the total sensing volume of a LSP sensor compared with a typical SPR sensor. The expected peak shift, induced by a molecular film with thickness $t$ and effective refractive index $n_{\mathrm{f}}$ can be obtained from $\lambda_{p}=\left(\frac{d \lambda}{d n}\right)_{b u l k} \cdot\left(n_{\text {film }}-n_{s}\left(1-\exp \left(-\frac{t}{L}\right)\right)\right.$ where $\left(\frac{d \lambda}{d n}\right)_{b u l k}$ is the bulk sensitivity and $n_{\mathrm{s}}$ is the refractive index of the surrounding medium and L is the decay length ${ }^{19}$. Thus the LSP will be affected more by the aptamer coating. Comparing these results with those given in reference 20 (detection limits in the order of $\sim 1 \mathrm{nM}$ ) this SPR device shows good detection sensitivity.

\section{Conclusion}

Surface plasmonic fibre devices fabricated with multiple coatings deposited on a lapped section of a single mode fibre, have suggested detection limits of sub-pM concentrations of thrombin in a sodium phosphate buffer solution, which would outperform other types of fibre optic thrombin sensors.

\section{References}

[1] D. Ellington, J. Szostak, "In vitro Selection of RNA Molecules that Bind Specific Ligands" Nature, Vol. 346, pp.818-822, 1990

[2] C. Tuerk, L. Gold, "Systematic evolution of ligands by exponential enrichment: RNA ligands to bacteriophage T4 DNA polymerase", Science, Vol. 249, pp.505-510. 1990

[3] N. O. Fischer, T. M. Tarasow, J. B. Tok, “Aptasensors for biosecurity applications” Curr. Opin. Chem. Biol., Vol. 11, pp.316-328. 2007

[4] G. Szakacs, et al, "Targeting multidrug resistance in cancer", Nat. ReV. Drug DiscoVery, Vol. 5, pp.219-234, 2006.

[5]Ramjee, M.K, "The use of fluorogenic substrates to monitor thrombin generation for the analysis of plasma and whole blood coagulation", Anal. Biochem. Vol. 277, pp.11-18. 1999.

[6] S. R. Garden, et al, "A fluorescent coagulation assay for thrombin using a fibre optic evanescent wave sensor", Biosensors and Bioelectronics, Vol. 19, pp.737-740, 2004

[7] A. Leung, P. M. Shankar, R. Mutharasan, "A review of fiber-optic biosensors", Sensors and Actuators B, Vol. 125, pp. 688-703, 2007

[8]T.M. Battaglia et al, "Quantification of cytokines involved in wound healing using surface plasmon resonance", Anal. Chem., Vol.77, No.21, pp.7016-7023. 2005

[9] J.F. Masson, et al, "Sensitive and real-time fiber-optic-based surface plasmon resonance sensors for myoglobin and cardiac troponin" I, Talanta Vol. 62, No. 5, pp.865-870. 2004

[10] S. Patskovsky et al, "Properties and sensing characteristics of surface plasmon resonance in infrared light", J. Opt. Soc. Am. A, Vol.20, No. 8, pp.1644-1650, 2003.

[11] M. Piliarik et al, "Surface plasmon resonance sensor based on a single-mode polarisation-maintaining optical fiber", Sensors and Actuators B, Vol. 90, pp.236-242, 2004

[12] R. Slavík, "Ultrahigh resolution long range surface plasmon-based sensor", Sensors and Actuators B, Vol.123, No. 1, pp.10-12, 2007.

[13] A. J. Haes et al, “A unified view of propagating and localized surface plasmon resonance biosensors", Anal. Bioanal. Chem. Vol. 379, pp.920, 2004

[14] "Surface Plasmons on smooth and Rough Surfaces and on Gratings", H. Raether,, eds. (Academic, New York, 1997),

[15] J. M. Brockman et al, "Surface Plasmon Resonance Imaging Measurement of Ultra-thin Organic Films", Annu. Rev. Phys. Chem., Vol. 51, pp.41-63, 2000.

[16] S. Tombelli, M. Minunni, M. Mascini, "A surface plasmon resonance biosensor for the determination of the affinity of drugs for nucleic acids", Anal. Lett., Vol.35, pp.599 - 613, 2002

[17] E. J. Cho, et al, "Applications of Aptamers as Sensors, Annual Review of Analytical Chemistry, Vol. 2, pp. 241-264, 2009

[18] E. Torres-Chavolla, E. C. Alocilja, "Aptasensors for detection of microbial and viral pathogens", Biosensors and Bioelectronics, Vol.24, pp.3175-3182, 2009

[19] M. P. Jonsson, et al, "Nanoplasmonic biosensing with focus on short-range ordered nanoholes in thin metal films: Review", Biointerphases Vol. 3, No. 3, pp.30-40

[20] X. Fan, et al, "Sensitive optical biosensors for unlabeled targets: A review", analytica chimica acta, Vol. 62 0, pp.8-26, 2008 